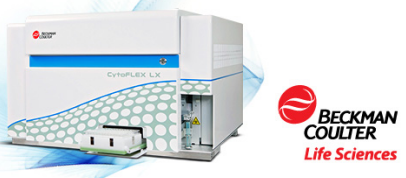

AA The Journal of Immunology

This information is current as of June 19, 2017.
Mapping the Binding Site of a Cross-Reactive Plasmodium falciparum PfEMP1 Monoclonal Antibody Inhibitory of ICAM-1 Binding

Frank Lennartz, Anja Bengtsson, Rebecca W. Olsen, Louise Joergensen, Alan Brown, Louise Remy, Petr Man, Eric Forest, Lea K. Barfod, Yvonne Adams, Matthew K. Higgins and Anja T. R. Jensen

J Immunol 2015; 195:3273-3283; Prepublished online 28 August 2015;

doi: 10.4049/jimmunol.1501404

http://www.jimmunol.org/content/195/7/3273
Supplementary Material

References

Subscription

Permissions

Email Alerts http://www.jimmunol.org/content/suppl/2015/08/28/jimmunol.150140 4.DCSupplemental

This article cites $\mathbf{4 0}$ articles, 13 of which you can access for free at: http://www.jimmunol.org/content/195/7/3273.full\#ref-list-1

Information about subscribing to The Journal of Immunology is online at: http://jimmunol.org/subscription

Submit copyright permission requests at: http://www.aai.org/About/Publications/JI/copyright.html

Receive free email-alerts when new articles cite this article. Sign up at: http://jimmunol.org/alerts 


\title{
Mapping the Binding Site of a Cross-Reactive Plasmodium falciparum PfEMP1 Monoclonal Antibody Inhibitory of ICAM-1 Binding
}

\author{
Frank Lennartz, ${ }^{*, 1}$ Anja Bengtsson, ${ }^{\dagger,+1}$ Rebecca W. Olsen, ${ }^{\dagger,+, 1}$ Louise Joergensen, ${ }^{\dagger, *}$ \\ Alan Brown, ${ }^{\S}$ Louise Remy, "Petr Man,",\# Eric Forest, ${ }^{\complement}$ Lea K. Barfod, ${ }^{\dagger, \ddagger}$ \\ Yvonne Adams, ${ }^{\dagger, *}$ Matthew K. Higgins, ${ }^{*}$ and Anja T. R. Jensen ${ }^{\dagger, *}$
}

The virulence of Plasmodium falciparum is linked to the ability of infected erythrocytes (IE) to adhere to the vascular endothelium, mediated by $P$. falciparum erythrocyte membrane protein 1 (PfEMP1). In this article, we report the functional characterization of an mAb that recognizes a panel of PfEMP1s and inhibits ICAM-1 binding. The 24E9 mouse mAb was raised against PFD1235w DBLß3_D4, a domain from the group A PfEMP1s associated with severe malaria. 24E9 recognizes native PfEMP1 expressed on the IE surface and shows cross-reactivity with and cross-inhibition of the ICAM-1 binding capacity of domain cassette 4 PfEMP1s. 24E9 Fab fragments bind DBLB3_D4 with nanomolar affinity and inhibit ICAM-1 binding of domain cassette 4-expressing IE. The antigenic regions targeted by $24 \mathrm{E} 9$ Fab were identified by hydrogen/deuterium exchange mass spectrometry and revealed three discrete peptides that are solvent protected in the complex. When mapped onto a homology model of DBLB3_D4, these cluster to a defined, surface-exposed region on the convex surface of DBLB3_D4. Mutagenesis confirmed that the site most strongly protected is necessary for 24E9 binding, which is consistent with a low-resolution structure of the DBLß3_D4::24E9 Fab complex derived from small-angle $x$-ray scattering. The convex surface of DBLB3_D4 has previously been shown to contain the ICAM-1 binding site of DBL及 domains, suggesting that the mAb acts by occluding the ICAM-1 binding surface. Conserved epitopes, such as those targeted by 24E9, are promising candidates for the inclusion in a vaccine interfering with ICAM-1-specific adhesion of group A PfEMP1 expressed by $P$. falciparum IE during severe malaria. The Journal of Immunology, 2015, 195: 3273-3283.

$\mathrm{H}$ uman malaria caused by Plasmodium falciparum parasites remains a serious health problem. In 2013, an estimated 198 million cases of malaria resulted in 584,000 deaths, mostly in sub-Saharan Africa (1). The majority of deaths occurred in children $<5$ y of age.

Parasite virulence is linked to the ability of infected erythrocytes (IE) to adhere to the inside of host blood vessels, leading to inflammation, tissue obstruction, and organ dysfunction (2). IE adhesion is mediated by the surface expression of $P$. falciparum erythrocyte membrane protein 1 (PfEMP1) proteins, which are able to bind to various host receptors present on the endothelium.

The multidomain PfEMP1 proteins are encoded by $\sim 60$ divergent var genes and consist of Duffy-binding-like (DBL) and cysteine-rich interdomain region protein domains (3), which can be divided into several major types $(\alpha, \beta, \gamma$, etc.) and subtypes based on sequence similarities $(4,5)$. DBL domains generally contain three subdomains, which fold together to form a conserved $\alpha$-helical core with loop insertions of variable sequence and length. Specific DBL and cysteine-rich interdomain region domains group together to form domain cassette (DC) families that are found across parasite isolates (5).

A frequently described PfEMP1 receptor is ICAM-1, and binding of IE to ICAM-1 during infection is linked to the development of symptoms of severe malaria, such as cerebral malaria (6-8). ICAM-1 is a membrane-bound protein with five extracellular domains (D1-D5) and is expressed by endothelial cells and leukocytes. ICAM-1 mediates leukocyte adhesion and migration to inflamed sites by binding to LFA-1 and Mac-1 $(9,10)$.
*Department of Biochemistry, University of Oxford, Oxford OX1 3QU, United Kingdom; Department of Immunology and Microbiology, Centre for Medical Parasitology, Faculty of Health and Medical Sciences, University of Copenhagen, Copenhagen 1014, Denmark; "Department of Infectious Diseases, Copenhagen University Hospital (Rigshospitalet), Copenhagen 2100, Denmark; ${ }^{\S}$ Department of Biochemistry, University of Cambridge, Cambridge CB2 1GA, United Kingdom; "Institut de Biologie Structurale, Grenoble F-38044, France; "Institute of Microbiology, Academy of Sciences of the Czech Republic, 11720 Prague, Czech Republic; and ${ }^{\#}$ Faculty of Science, Charles University in Prague, 11636 Prague, Czech Republic

${ }^{1}$ F.L., A.B., and R.W.O. contributed equally to this work.

ORCID: 0000-0002-4004-7554 (A.T.R.J.).

Received for publication June 23, 2015. Accepted for publication July 23, 2015.

M.K.H. was supported by a Wellcome Trust investigator Award. F.L. and M.K.H were supported by a project grant from the Medical Research Council (Grant G0901062). A.B., R.W.O., L.J., L.K.B., and A.T.R.J. were supported by grants from the Faculty of Health and Medical Sciences, the University of Copenhagen, the Aase and Ejnar Danielsens Fond, the Dagmar Marshalls Fond, the Oda and Hans Svenningsens Fond, and the Novo Nordisk Fonden. Y.A. was supported by the Danish Council for Independent Research (Grant 4004-00032). P.M. was supported by European Union Grants CZ.2.16/3.1.00/24023 and CZ.1.05/1.1. 00/02.0109.

The nucleotide sequences presented in this article have been submitted to GenBank (http:/ www.ncbi.nlm.nih.gov/genbank) under accession numbers KJ418726 and KJ418727.

Address correspondence and reprint requests to Prof. Matthew K. Higgins or Prof. Anja T.R. Jensen, Department of Biochemistry, University of Oxford, South Parks Road, Oxford OX1 3QU, U.K. (M.K.H.) or Department of Immunology and Microbiology, Faculty of Health and Medical Sciences, University of Copenhagen, CSS-Building 22, Oster Farimagsgade 5, Copenhagen K, 1014, Denmark (A.T.R.J.). E-mail addresses: matthew.higgins@bioch.ox.ac.uk (M.K.H.) or atrj@sund.ku.dk (A.T.R.J.)

The online version of this article contains supplemental material.

Abbreviations used in this article: DBL, Duffy-binding-like; DC4, domain cassette 4; $\mathrm{D}_{\max }$, maximum particle diameter; HDX MS, hydrogen/deuterium exchange mass spectrometry; IE, infected erythrocyte; PDB, Protein Data Bank; PfEMP1, P. falciparum erythrocyte membrane protein 1 ; Rg, radius of gyration; RU, response unit; SAXS, small-angle x-ray scattering; SPR, surface plasmon resonance.

This is an open-access article distributed under the terms of the CC-BY 3.0 Unported license. 
Surface expression of the recently identified DC4 containing PfEMP1s leads to ICAM-1-specific adhesion of IE, which is mediated by the DBL 33 D4 PfEMP1 domain $(11,12)$ and appears to be involved in the pathogenesis of severe disease (13). Naturally acquired Abs against DC4 DBL 33 _D4 are cross-reactive and crossinhibitory of ICAM-1 binding across members of DC4 and other DC types (12), suggesting that the DC4 DBL $\beta 3$ domains are attractive vaccine candidates.

Although no crystal structure exists currently for a DBL $\beta$ :: ICAM-1 complex, this interaction has been studied in a number of different ways. Studies with truncated or mutated ICAM-1 constructs show that the binding site for DBL $\beta$ domains locates to the D1 domain of ICAM-1, and experiments with truncated and chimeric proteins have mapped the ICAM-1 binding site to the C-terminal end of DBL $\beta$ (14-17). In addition, ICAM-1 binding is gained when replacing the $\mathrm{C}$-terminal subdomain of an ICAM-1 nonbinding DBL $\beta 3$ with that of the ICAM-1 binding PFD1235w DBLB3_D4 (12). Homology modeling (18) and smallangle $\mathrm{x}$-ray scattering (SAXS) (19), together with mutagenesis studies (20), further suggest that the interaction surface is on the convex surface of the DBL $\beta$ domain. However, the exact amino acids involved in DBL $\beta$ binding to ICAM- 1 are yet to be determined.

The identification of DBL $\beta$ region(s) targeted by protective Abs and a detailed mapping of ICAM-1 binding epitopes will be an essential step toward designing a PfEMP1-based vaccine potentially protective against malaria. Using modern methods for the characterization of $\mathrm{Ab}-\mathrm{Ag}$ complexes, such as hydrogen/deuterium exchange mass spectrometry (HDX MS), surface plasmon resonance (SPR), and SAXS, we characterized an mAb (24E9) that binds to the convex surface of DC4 DBL 33 _D4 domains and interferes with the DBL $\beta$ ::ICAM-1 interaction. We show that 24E9 $\mathrm{mAb}$ targets epitopes conserved between DC4 DBL $\beta$ domains from genetically distant parasite isolates and inhibits ICAM-1 binding of IE by blocking the predicted ICAM-1 binding site on DBL $\beta$. This provides important knowledge for choosing components for a vaccine aimed at preventing PfEMP1-mediated adhesion of IE during severe malaria.

\section{Materials and Methods}

\section{Recombinant protein expression and purification}

Full-length, wild-type PFD1235w DBLß3_D4 was subcloned into a modified pET15b vector and expressed as an $\mathrm{N}$-terminal, hexahistidine-tagged protein in Escherichia coli SHuffle 3030 cells (New England Biolabs) for $16 \mathrm{~h}$ at $25^{\circ} \mathrm{C}$. The cells were pelleted, washed, and lysed, and DBLB3_D4 was purified using Ni-NTA-Sepharose (QIAGEN). The hexahistidine-tag was removed by overnight cleavage at $4^{\circ} \mathrm{C}$ using Tobacco etch virus protease. Tobacco etch virus protease and uncleaved protein were removed by reverse immobilized-metal affinity chromatography, and DBL 33 _D4 was further purified by size exclusion chromatography using a Superdex 75 16/60 column (GE Healthcare).

PFD1235w DBLB3_D4 protein used for mouse immunization to generate hybridomas was subjected to an additional purification step. DBLB3_D4 was allowed to bind to ICAM-1_D1-D5-Fc coupled to a HiTrap NHS-activated HP column (GE Healthcare). Bound DBL 33 D4 was eluted from ICAM- 1 on the column and buffer exchanged into PBS.

For generation of DBL 33 _D4 mutants, a set of 5' phosphorylated primers that included the coding sequence for the P2b or P3a regions of DBLß3_D5 was used to amplify the DBLß3_D4-encoding pEt15b vector by PCR. The PCR products were circularized by blunt-end ligation using T4 ligase (Life Technologies), and the mutants were expressed and purified as described for DBLB3_D4.

ICAM-1 domains 1-5 (D1-D5) combined with the Fc region of human IgG1 (ICAM-1-Fc) was cloned, expressed, and purified as described previously (21). ICAM-1_D1-D2 was expressed in COS-7 cells and purified as described previously (19). ICAM-1_D1 was expressed and purified from E. coli BL21(DE3) as described previously (22).

\section{CD spectroscopy}

Far-UV CD spectroscopy experiments were carried out with a J-815 Spectropolarimeter (Jasco) equipped with a computer-controlled Peltier temperature control unit. All samples were dialyzed into $10 \mathrm{mM}$ sodium phosphate buffer, $150 \mathrm{mM} \mathrm{NaF}, \mathrm{pH} 7.2$, and measurements were taken at a protein concentration of $0.1 \mathrm{mg} / \mathrm{ml}$ using a $1 \mathrm{mM}$ path cell. Spectra were acquired at $20^{\circ} \mathrm{C}$ at wavelengths between 195 and $260 \mathrm{~nm}$. For thermal unfolding, the temperature was raised from $20^{\circ}$ to $95^{\circ} \mathrm{C}$ in $0.5^{\circ} \mathrm{C}$ increments, and spectra were recorded between 200 and $250 \mathrm{~nm}$ wavelength.

\section{Hybridoma production}

24E9 hybridomas were produced according to standard protocols (23). One CB6FI mouse (Harlan) was immunized s.c. with $30 \mu \mathrm{g}$ PFD1235w DBLB3_D4 in CFA (Sigma-Aldrich) followed by two additional boosters of $15 \mu \mathrm{g}$ protein in IFA (Sigma-Aldrich). A final i.v. boost of $15 \mu \mathrm{g}$ protein in PBS was given $3 \mathrm{~d}$ before the mouse was sacrificed and the spleen was taken out. Single spleen B lymphocytes were made from the whole spleen and fused to SP2/0-AG14 Myeloma cells (ATCC) in a 1:2 ratio using polyethylene glycol 4000 . Spleen and myeloma cell mixture was diluted in $80 \mathrm{ml}$ cell media (RPMI, 20\% FBS, glutamine, penicillin/streptomycin) containing HAT media supplement (Sigma-Aldrich) to select for fused cells. A total of $100 \mu 1 /$ well was added to eight deep, flat-bottom, 96-well plates (Fisher Scientific) containing peritoneal macrophages from two $\mathrm{BALB} / \mathrm{C}$ mice (Taconic) serving as feeder cells. Cells were grown for $1 \mathrm{wk}$ at $37^{\circ} \mathrm{C}, 5 \% \mathrm{CO}_{2}$ before changing the cell supernatant to cell media supplemented with HT media supplement (hypoxanthine and thymidine; SigmaAldrich). Two weeks after fusion, wells with growing cells were identified under a microscope and the cells were moved into fresh 96-well plates. After $1 \mathrm{wk}$, undiluted cell supernatant from each well was tested for the presence of DBLB3_D4-reactive Abs using ELISA. To obtain true monoclonal hybridomas, we cloned cells from positive wells by limiting dilution. All animal procedures were approved by the Danish National Committee (Dyreforsøgstilsynet) in agreement with permit no. 2008/561-1498.

\section{$m A b$ purification}

24E9 monoclonal hybridomas were expanded and seeded at $\sim 10 \%$ confluency in $175-\mathrm{cm}^{2}$ cell flasks containing $70 \mathrm{ml}$ cell media [RPMI, $10 \%$ low IgG FBS (Lonza), HT media supplement (Sigma-Aldrich), glutamine, penicillin/streptomycin]. After incubation for $1 \mathrm{wk}$ at $37^{\circ} \mathrm{C}, 5 \% \mathrm{CO}_{2}$ cell supernatant was centrifuged, sterile-filtered, and buffer-exchanged into PBS before purifying mAb using a HiTrap protein G column (GE Healthcare) according to the manufacturer's instructions.

\section{IgG subtyping}

The IgG subtype and $\mathrm{L}$ chain class of $24 \mathrm{E} 9 \mathrm{mAb}$ were determined using an IsoQuick Kit for Mouse Monoclonal Isotyping (Sigma) according to the manufacturer's instructions.

\section{Fab fragmentation}

Purified 24E9 mAb was buffer-exchanged into cleavage buffer $(0.1 \mathrm{M}$ sodium phosphate $\mathrm{pH} 6.4,0.3 \mathrm{M} \mathrm{NaCl}, 2 \mathrm{mM}$ EDTA, $5 \mathrm{mM}$ L-cysteine, $1.5 \mathrm{mM}$ 2-ME) and concentrated to $1 \mathrm{mg} / \mathrm{ml}$. Papain-agarose (SigmaAldrich) was added in a $20: 1$ ratio and incubated overnight at $37^{\circ} \mathrm{C}$. Papain-agarose was removed by centrifugation, and the $\mathrm{Fc}$ portion and uncleaved $\mathrm{mAb}$ were removed from the supernatant by purification on a protein A column (GE Healthcare). Fab fragments in the flow-through were further purified by size exclusion chromatography.

\section{Western blot}

Purified 24E9 mAb was tested for reactivity against reduced (+DTT) and nonreduced (-DTT) PFD1235w DBL 33 _D4. Purified PFD1235w DBLB3_D4 and PFD1235w DBLß3_D5 (control; $0.5 \mu \mathrm{g}$ ) were separated by SDS-PAGE under both conditions on a NuPAGE Novex $4-12 \%$ BisTris gel in MOPS SDS Running buffer (Invitrogen) and subsequently blotted onto a Hybond-C Extra NC membrane (GE Healthcare). The membrane was blocked using $2.5 \%$ skimmed milk in dilution buffer (PBS, $1 \% \mathrm{BSA}$ ). The 24E9 mAb was diluted to $10 \mu \mathrm{g} / \mathrm{ml}$ in dilution buffer and added to the membrane. Bound 24E9 mAb was detected by anti-mouse IgG (P260; Dako) 1:1000 in dilution buffer using a chemiluminescent detection kit (Thermo Scientific).

\section{ELISA}

Hybridoma screening. Hybridoma cell supernatants were screened for PFD1235w DBL33_D4-reactive Abs using ELISA. Duplicate wells of 
MaxiSorp microtiter plates (Nunc) were coated with DBLB3_D4 (50 $\mu \mathrm{l}$; $1 \mu \mathrm{g} / \mathrm{ml} ; 0.1 \mathrm{M}$ glycine/ $\mathrm{HCl}$ buffer $\mathrm{pH} 2.75$; overnight; $4^{\circ} \mathrm{C}$ ) and blocked with blocking buffer (PBS, $0.5 \mathrm{M} \mathrm{NaCl}, 1 \%$ Triton X-100, $1 \%$ BSA, pH 7.2). A total of $100 \mu \mathrm{l}$ undiluted cell supernatant was added $(1 \mathrm{~h}$; room temperature). The plates were washed in PBS $+1 \%$ Triton X-100, and bound $\mathrm{Ab}$ was detected with an anti-mouse Ig-HRP (Dako; 1:3000 in blocking buffer). After $1 \mathrm{~h}$ of incubation, plates were developed using OPD tablets (Dako) according to the manufacturer's instructions. The OD value was read at $490 \mathrm{~nm}$ using a VERSAmax microplate reader (Molecular Devices) and Softmax Pro v4.7.1.

$m A b$ reactivity. Microtiter plates were coated with $50 \mu 1,2 \mu \mathrm{g} / \mathrm{ml}$ recombinant proteins in glycine/ $\mathrm{HCl}$ buffer and blocked with blocking buffer. 24E9 mAb (50 $\mu$ l; 3-fold dilutions starting at $10 \mu \mathrm{g} / \mathrm{ml}$; $1 \mathrm{~h}$; room temperature) was added, and washing was performed as described above. Bound $\mathrm{Ab}$ was detected with anti-mouse Ig-HRP (Dako; 1:3000 in blocking buffer; $1 \mathrm{~h}$; room temperature).

Reducing ELISA. Microtiter plates were coated (50 $\mu \mathrm{l} ; 2$-fold dilutions starting at $64 \mu \mathrm{g} / \mathrm{ml}$; glycine/ $\mathrm{HCl}$ buffer; overnight; $4^{\circ} \mathrm{C}$ ) with $24 \mathrm{E} 9 \mathrm{mAb}$ or the PFD1235w DBL $\gamma$-specific AB01 mAb (24) and blocked with PBS + $1 \%$ BSA. Plates were washed in PBS and PFD1235w DBLB3_D4 or PFD1235w DBL $\gamma(50 \mu \mathrm{l} ; 2 \mu \mathrm{g} / \mathrm{ml}$; PBS $\pm 50 \mathrm{mM}$ DTT; $1 \mathrm{~h}$; room temperature) were added to the plates coated with 24E9 or AB01, respectively. Bound DBL $\beta 3$ _D4 or DBL $\gamma$ was detected by use of an antipenta-His $\mathrm{HRP} \mathrm{Ab}(1: 3000$ in PBS $+1 \% \mathrm{BSA} ; 1 \mathrm{~h}$; room temperature; QIAGEN). Washing and detection were performed as described above.

ICAM-1 inhibition ELISA. Microtiter plates were coated with recombinant ICAM-1-Fc $\left(50 \mu \mathrm{l}, 2 \mu \mathrm{g} / \mathrm{ml}\right.$; glycine/HCl buffer; overnight; $\left.4^{\circ} \mathrm{C}\right)$ and blocked with blocking buffer. DBL 33 D4 domains $(1-16 \mu \mathrm{g} / \mathrm{ml})$ were added simultaneously with mAb 24E9 added in 2-fold dilutions ranging from 0.25 to $32 \mu \mathrm{g} / \mathrm{ml}$. Mouse IgG (Life Technologies) was added as control. ICAM-1-bound DBLB3_D4 was detected using anti-penta-His $\mathrm{HRP} \mathrm{Ab}$ (1:3000 in blocking buffer; $1 \mathrm{~h}$; room temperature; QIAGEN). Washing and detection were performed as described above.

\section{Sequencing}

The mouse Ig L and $\mathrm{H}$ chain variable genes of the $24 \mathrm{E} 9 \mathrm{mAb}$ were sequenced to determine the amino acid sequences of the CDRs. cDNA was made from single 24E9 hybridoma cells using a QIAGEN OneStep RT-PCR Kit with degenerate primers designed to target mouse Ig variable regions (25). cDNA was amplified using Phusion HF polymerase (New England Biolabs), and PCR products were sequenced using BigDye Terminator v3.1 Cycle Sequencing Kit (Applied Biosystems) according to the manufacturer's instructions. Sequence data were collected on a 3100-Avant Genetic Analyzer (Applied Biosystems). The nucleotide sequences of 24E9 CDRs can be retrieved from GenBank using accession numbers KJ418726 (H chain) and KJ418727 (L chain) (http://www.ncbi.nlm.nih. gov/genbank).

\section{Malaria parasites and flow-cytometry analysis}

The 3D7 P. falciparum clone and one Ghanaian patient isolate (BM057) were cultured in vitro (26) and were selected for DC4 PfEMP1 IE surface expression by repeated $\mathrm{Ab}$ selection as described previously (12). The identity of the isolates was routinely verified by genotyping as described previously (27), and Mycoplasma infection was regularly excluded using the MycoAlert Mycoplasma Detection Kit (Lonza) according to the manufacturer's instructions.

P. falciparum IE were DNA-labeled with ethidium bromide and surfacelabeled with mouse antisera obtained from the immunized mouse used for hybridoma production $(15 \mu \mathrm{l}$ serum/well $), 24 \mathrm{E} 9 \mathrm{mAb}(100 \mu \mathrm{g} / \mathrm{ml})$, or 24E9 Fab fragments $(100 \mu \mathrm{g} / \mathrm{ml})$. Whole Abs were labeled using an FITCconjugated secondary anti-mouse IgG (1:100; Vector Labs), and an antimouse $\mathrm{F}\left(\mathrm{ab}^{\prime}\right)_{2}$ IgG (1:100; Jackson Immunoresearch) was used to detect Fab fragments. FITC fluorescence data from ethidium bromide ${ }^{+}$cells were collected on a Cytomics FC 500 MPL flow cytometer (Beckman Coulter) and analyzed in WinList version 6.0 (Verity Software House).

\section{ICAM-1 adhesion assays under physiological flow conditions}

Biochips (Vena8; Cellix) were coated at $4^{\circ} \mathrm{C}$ overnight with recombinant ICAM-1-Fc $(50 \mu \mathrm{g} / \mathrm{ml})$ produced as described previously (21). Channels $[400 \times 100 \times 20 \mathrm{~mm}(\mathrm{w} \times \mathrm{d} \times 1)]$ were blocked for $1 \mathrm{~h}$ at $37^{\circ} \mathrm{C}$ with PBS $+1 \%$ BSA and the chip mounted onto a Leica inverted phase-contrast microscope. To generate a wall shear stress representing that within microvasculature $\left(1 \mathrm{dyn} / \mathrm{cm}^{2}\right)$, we connected the biochip to an NE-1002X microfluidic pump (World Precision Instruments, U.K.). Erythrocytes at $3-$ $5 \%$ parasitemia (1\% hematocrit in RPMI 1640 plus $2 \%$ normal human serum) were flowed over the biochip for $5 \mathrm{~min}$. The number of bound IE per square millimeter for five separate fields was counted at 20 times magnification, and a minimum of three independent experiments was done in triplicates.

To inhibit ICAM-1 adhesion, we combined IE with $24 \mathrm{E} 9 \mathrm{mAb}(1 \times$ $\left.10^{-1} ; 1,10 \mu \mathrm{g} / \mathrm{ml}\right)$ or Fab fragments of $24 \mathrm{E} 9\left(1 \times 10^{-3} ; 1 \times 10^{-1}\right.$; $1 \mu \mathrm{g} / \mathrm{ml})$ before assaying as described earlier. Mouse IgG $(10 \mu \mathrm{g} / \mathrm{ml}$; Life Technologies) or mouse IgG Fab fragments ( $1 \mu \mathrm{g} / \mathrm{ml}$; Rockland) were included as negative controls. Specificity of adhesion to recombinant ICAM-1-Fc was determined by the preincubation of channels with $40 \mu \mathrm{g} / \mathrm{ml}$ anti-ICAM-1 (clone 15.2; AbD Serotec).

\section{$S P R$}

SPR measurements were conducted using a BIAcore T-100 instrument (GE Healthcare). DBLB3_D4 was diluted into $10 \mathrm{mM}$ acetate buffer, $\mathrm{pH} 4.0$, and covalently coupled to a CM5 chip (GE Healthcare) by amine coupling to a density of 400 response units (RU). ICAM-1_D1, ICAM-1_D1-D2, and $\mathrm{mAb} 24 \mathrm{E} 9 \mathrm{Fab}$ were prepared in $10 \mathrm{mM}$ HEPES $\mathrm{pH} 7.2,150 \mathrm{mM}$ $\mathrm{NaCl}, 50 \mu \mathrm{M}$ EDTA, and $0.05 \%$ Tween 20 . For each protein, a concentration series $(100,50,25,12.5$, and $6.25 \mu \mathrm{g} / \mathrm{ml})$ was flowed over the chip surface at a flow rate of $45 \mu \mathrm{l} / \mathrm{min}$ with an association time of $120 \mathrm{~s}$ and a dissociation time of $400 \mathrm{~s}$. The signal from an empty flow cell was subtracted from all measurements. Between runs, the sensor surface was regenerated with $4 \mathrm{M} \mathrm{MgCl}_{2}$ for $30 \mathrm{~s}$ at a flow rate of $30 \mu \mathrm{l} / \mathrm{min}$ for the 24E9 Fab::DBLß3_D4 interaction, or $5 \mathrm{mM} \mathrm{NaOH}$ for $10 \mathrm{~s}$ at a flow rate of $30 \mu \mathrm{l} / \mathrm{min}$ for the ICAM-1_D1::DBL 33 D4 and ICAM-1_D1-D2:: DBL $\beta 3$ D4 interactions.

For analysis of DBLB3_D4 mutant binding to 24E9 mAb, 24E9 mAb was immobilized to $230 \mathrm{RU}$ on a CM5 chip (GE Healthcare) precoupled with protein G. DBL $\beta 3$ _D4 mutants were diluted into $10 \mathrm{mM}$ HEPES $\mathrm{pH}$ $7.2,300 \mathrm{mM} \mathrm{NaCl}$, and $0.05 \%$ Tween 20 , and for each mutant a concentration series $(31.25,15.62,7.81,3.90,1.95$, and $0.97 \mathrm{nM})$ was flowed over the chip surface at $40 \mu \mathrm{l} / \mathrm{min}$ with $240 \mathrm{~s}$ association time and $400 \mathrm{~s}$ dissociation time. The signal from a flow cell lacking the DBLB3_D4 domain was subtracted from all measurements. The sensor surface was regenerated between runs with $100 \mathrm{mM}$ glycine- $\mathrm{HCl}, \mathrm{pH} 2.0$ for $120 \mathrm{~s}$ at a flow rate of $10 \mu \mathrm{l} / \mathrm{min}$

For all measurements, sensorgrams corresponding to at least four different concentrations were globally fitted into a one-site kinetic model, and the values for $k_{\mathrm{a}}, k_{\mathrm{d}}$, and $K_{\mathrm{D}}$ were obtained using the BIAevaluation software 2.0.3 (GE Healthcare).

\section{$H D X M S$}

HDX MS experiments were fully automated using a PAL autosampler (CTC Analytics). This controlled the start of exchange and quench reactions, proteolysis temperature $\left(4^{\circ} \mathrm{C}\right)$, injection of the deuterated peptides, management of the injection and washing valves, and triggering of HPLC pumps and acquisition by the mass spectrometer. A Peltier-cooled box $\left(4^{\circ} \mathrm{C}\right)$ contained two Rheodyne automated valves (6-port for injection and 10-port for washing), a desalting cartridge (peptide Opti-Trap Micro from Optimize Technologies), and an HPLC column (C18 Jupiter $4 \mu \mathrm{m}$ Proteo $90 \AA$ A, $50 \times 1 \mathrm{~mm}$ from Phenomenex). HDX MS reactions were carried out using gel-filtered DBL 33 D4 and DBLß3_D4::24E9 Fab (1:1 molar ratio), both at concentrations of $40 \mu \mathrm{M}$. Deuteration was initiated by a 5 -fold dilution of DBL33_D4 or DBL33_D4::24E9 Fab (10 $\mu$ l) with PBS in $\mathrm{D}_{2} \mathrm{O}$ $(40 \mu \mathrm{l})$. The proteins were deuterated for $20 \mathrm{~min}$ at $4^{\circ} \mathrm{C}$ or $20 \mathrm{~min}$ at room temperature $\left(26^{\circ} \mathrm{C}\right)$. Considering the change of exchange kinetics of amide hydrogens with temperature (about a 3-fold exchange increase for each $10^{\circ} \mathrm{C}$ increase in temperature), the last condition is equivalent to a $200-\mathrm{min}$ deuteration at $4^{\circ} \mathrm{C}$. A total of $50 \mu \mathrm{l} 0.8 \mathrm{M}$ Tris(2-carboxyethyl)phosphine, $2 \mathrm{M}$ glycine was added for $10 \mathrm{~min}$ at $4^{\circ} \mathrm{C}$ to quench back-exchange and to reduce disulphide bridges. The proteins were digested online with immobilized porcine pepsin (Sigma) and recombinant nepenthesin-1 (28) proteases. The peptides were desalted using an HPLC pump (Agilent Technologies) with $0.03 \%$ trifluoroacetic acid in water (buffer A) at a flow rate of $100 \mu \mathrm{l} / \mathrm{min}$. The peptides were then separated using another HPLC pump (Agilent Technologies) at $50 \mu \mathrm{l} / \mathrm{min}$ for $6 \mathrm{~min}$ with a $15-50 \%$ gradient of buffer B (buffer B: acetonitrile $90 \%$, trifluoroacetic acid $0.03 \%$ in water), followed by $9 \mathrm{~min}$ at $50 \% \mathrm{~B}$ and $5 \mathrm{~min}$ at $100 \% \mathrm{~B}$. The peptide masses were measured using an electrospray-time of flight mass spectrometer (Agilent 6210) in the $300-1300 \mathrm{~m} / \mathrm{z}$ range. The peptides were previously identified by tandem mass spectrometry, using a Bruker APEXQ FTMS (9.4 T). The Mass Hunter (Agilent Technologies) and Data Analysis (Bruker) software were used for data acquisition. The HD Examiner software (Sierra Analytics) was used for HDX MS data processing. For each deuteration time $\left(20 \mathrm{~min}\right.$ at $4{ }^{\circ} \mathrm{C}$ or $20 \mathrm{~min}$ at room temperature), experiments were performed in triplicate and measurements were averaged. 


\section{SAXS}

SAXS measurements were carried out at the EMBL BioSAXS P12 beamline at the DORIS storage ring, DESY (Hamburg, Germany). Scattering data were recorded at a wavelength of $1.24 \AA$ using a twodimensional photon counting PILATUS 2 million pixel x-ray detector (Dectris, Baden, Switzerland). The distance between detector and sample was $3.1 \mathrm{~m}$, resulting in a $\mathrm{q}$ range of $0.01-0.44 \AA^{-1}\left[\mathrm{q}=4 \pi \sin (\theta) \lambda^{-1}\right.$, where $\mathrm{q}$ is the scattering vector, $2 \theta$ is the scattering angle, and $\lambda$ is the wavelength].

Samples for SAXS were prepared in buffer containing $20 \mathrm{mM}$ HEPES, $150 \mathrm{mM} \mathrm{NaCl}, \mathrm{pH} 7.4$ and purified by size exclusion chromatography. Sample purity was verified by SDS-PAGE, and only samples with a purity $>95 \%$ were used for data collection. Before measurement, samples were centrifuged at $13,000 \mathrm{rpm}$ for $5 \mathrm{~min}$ at $4^{\circ} \mathrm{C}$. For each sample, a concentration series $(3.78,1.79,0.87,0.34,0.18 \mathrm{mg} / \mathrm{ml}$ for DBLB3_D4 and 4.64, $2.38,1.16,0.59,0.19 \mathrm{mg} / \mathrm{ml}$ for DBLB3_D4::24E9 Fab) was measured at $10^{\circ} \mathrm{C}$. Before and after each sample, buffer was measured as a control.

The scattering curves were manually inspected using PRIMUS (29), and frames showing signs of radiation damage were omitted in data analysis. Unaffected frames were averaged for each measurement, and the buffer signal was subtracted from the sample signal. To eliminate the effects of potential concentration-dependent protein aggregation at low scattering angles, the scattering curves of each concentration series were extrapolated to zero concentration. A composite curve was generated by scaling and merging the zero concentration curve with data for the highest concentration. The radius of gyration $(\mathrm{Rg})$ was estimated by Guinier analysis using AutoRg in PRIMUS, whereas the maximum particle diameter $\left(D_{\max }\right)$ and the pair distance distribution functions $\mathrm{P}_{\mathrm{r}}$ were calculated using GNOM (30).

$\mathrm{Ab}$ initio models were generated from solution scattering data by DAMMIF (31) using default parameters with P1 symmetry. For both DBLB3_D4 and DBLB3_D4::24E9 Fab, 20 independent DAMMIF models were averaged using DAMAVER (32). The averaged model was further refined by DAMMIN (33), using default parameters and the original pair distance distribution functions as input. SITUS was used to calculate volumetric representation from the bead models generated by DAMMIN, and homology models of DBLB3_D4 and a mouse Fab fragment (Protein Data Bank [PDB] ID 3GK8) were docked into the resulting envelopes using the program SCULPTOR (34). The DBLB3_D4 homology model was generated with I-TASSER (c-score -0.9 ) using the structures of DBL3X (PDB ID 3BQK), NTS-DBL1 $\alpha$ (PDB ID 2XU0), EBA-175 (PDB ID 1ZRL), and EBA-140 (PDB ID 4GF2) as templates. Structural models were visualized using PyMol Version 1.5.0.4 (Schrödinger).

\section{Results}

The 24E9 $\mathrm{mAb}$ is cross-reactive against DC4-containing PfEMP1 present on the surface of IE

We have previously observed that 3D7 PFD1235w DBLß3_D4 elicits adhesion-inhibitory Abs that are cross-reactive to DC4containing PfEMP1 from genetically distant parasite isolates (12). To study the specific epitopes targeted by such protective Abs in more detail, we first raised a monoclonal mouse Ab against 3D7 PFD1235w DBLß3_D4. This mAb, named 24E9, is of IgG1 isotype with $\mathrm{K} \mathrm{L}$ chains (data not shown). We tested the reactivity of 24E9 against native PfEMP1 on the surface of IE by flow cytometry. Both $24 \mathrm{E} 9 \mathrm{mAb}$ and Fab fragments generated from this $\mathrm{Ab}$ bound to erythrocytes infected with 3D7 parasites expressing DC4-containing PfEMP1 (Fig. 1). In contrast, 24E9 mAb did not recognize $\mathrm{DC}^{-}$3D7 IE.

24E9 mAb showed cross-reactivity to erythrocytes infected with the heterologous parasite strain BM057 (Fig. 1), which expresses a DC4 containing PfEMP1. We therefore used ELISA to test whether 24E9 mAb binds to other DBL $\beta$ domains. 24E9 mAb cross-reacted with five DBL 33 _D4 domains from DC4 containing PfEMP1 proteins cloned and expressed from Ghanaian field isolates (12) (Fig. 2A), but not with non-DC4 DBL $\beta$ domains from the IT4 isolate (Fig. 2B) or other PfEMP1 domains from the 3D7, Dd2, or HB3 isolates (Fig. 2C). Therefore, 24E9 mAb can recognize native PfEMP1 expressed on the surface of IE and shows patterns of cross-reactivity to DC4-containing PfEMP1s similar to
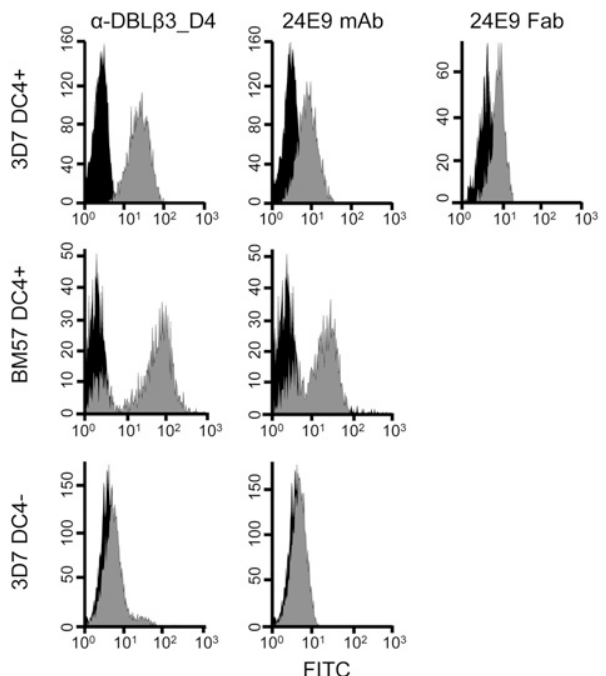

FIGURE 1. DC4-expressing $P$. falciparum IE are recognized by 24E9 $\mathrm{mAb}$ and Fab. PFD1235w DBLß3_D4 mouse anti-sera and 24E9 mAb were tested by flow cytometry on 3D7 DC4 $4^{+}, \mathrm{BM}_{7} 7 \mathrm{DC} 4^{+}$, and 3D7 DC4 ${ }^{-}$ parasite lines. 24E9 Fab was tested only on $\mathrm{DC}^{+} 3 \mathrm{D} 7$. IE with antisera, $24 \mathrm{E} 9 \mathrm{mAb}$, or 24E9 Fab are shown in gray, whereas IE without Abs (negative controls) are shown in black.

Abs found in pooled immune plasma from patients infected with parasites expressing 3D7 PFD1235w (12).

\section{The interaction between DC4 DBLB3_D4 and ICAM-1 is inhibited by $24 E 9$}

Binding to ICAM-1 is mediated through the DBL 33 _D4 domains of DC4 PfEMP1 (12). We therefore tested whether the 24E9 $\mathrm{mAb}$ blocks this interaction. We first compared the affinity of DBLB3_D4 for both 24E9 and ICAM-1 by SPR. We used 24E9 Fab fragments, leading to monovalent binding, which allowed for global fitting of the data with a one-site binding model. 24E9 Fab bound to DBLB3_D4 with low nanomolar affinity, comparable with the affinity of DBLB3_D4 for ICAM-1_D1-D2 and ICAM-1_D1 (Fig. 3A, Table I). Furthermore, the interaction between 24E9 Fab and DBL 33 _D4 showed fast association and slow dissociation rates similar to those observed for the interaction between ICAM-1_D1 and DBLB3_D4 (Fig. 3A-C, Table I).

We next analyzed whether 24E9 mAb directly inhibits the DBLß3_D4::ICAM-1 interaction. A chip coupled with DBLß3_D4 was preincubated with different concentrations of 24E9 Fab, followed by injection of ICAM-1_D1. As a control, ICAM-1_D1 was flowed over the chip surface without prior incubation with 24E9 Fab (Fig. 3D, red curve). Preabsorption of DBL 33 _D4 with increasing concentrations of 24E9 Fab reduced the binding of ICAM-1_D1 in a concentration-dependent manner (Fig. 3D, 3E), demonstrating that 24E9 Fab effectively blocks the interaction between DBLB3_D4 and ICAM-1_D1.

The observation that 24E9 is cross-reactive against several DC4 DBLB3_D4 domains from different parasite isolates (Fig. 2) raised the possibility that $24 \mathrm{E} 9$ also cross-inhibits the interaction between ICAM-1 and these domains. We tested this by ELISA and found that 24E9 mAb inhibited ICAM-1 binding of PFD1235w DBLß3_D4 and of the five DC4 DBLß3_D4 domains in a concentration-dependent manner (Fig. 3F). Taken together, these data show that $24 \mathrm{E} 9 \mathrm{mAb}$ is both cross-reactive and cross-inhibitory of ICAM-1 binding to all tested DC4 DBLB3_D4 domains and binds with a sufficiently strong affinity to effectively compete with ICAM-1 binding. 
FIGURE 2. 24E9 mAb is crossreactive against DC4 DBLB3_D4 domains. 24E9 $\mathrm{mAb}$ was tested against DC4-DBLB3_D4 domains (A), nonDC4 DBL $\beta$ domains from the IT4 isolate (B) and non-DC4 domains from 3D7, Dd2, and HB3 isolates (C) using ELISA. Mean OD values are shown for three independent experiments. Error bars indicate SD.
A
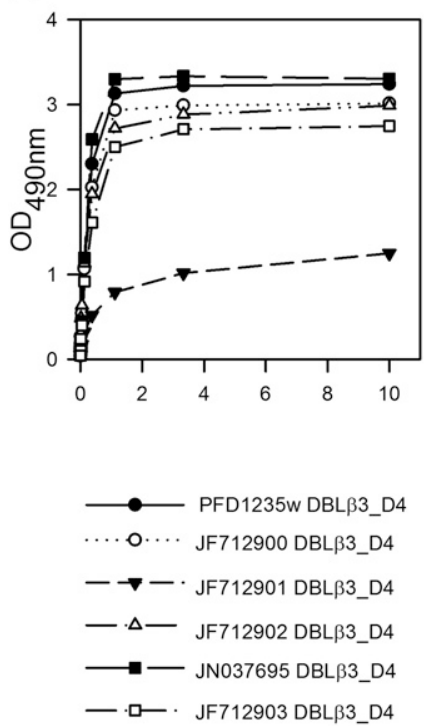

B

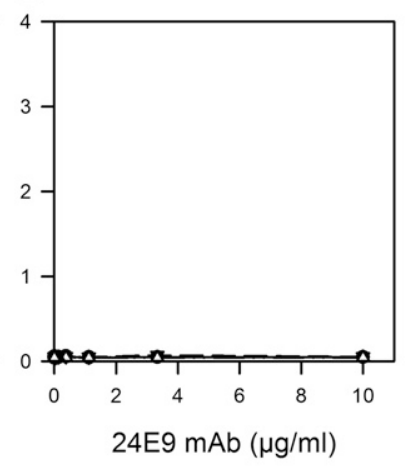

$\longrightarrow$ IT4var02 DBL $\beta 9$

… o.... IT4var13 DBLB5

$\longrightarrow \rightarrow-\cdot$ IT4var16 DBLB5

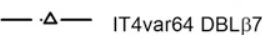

C

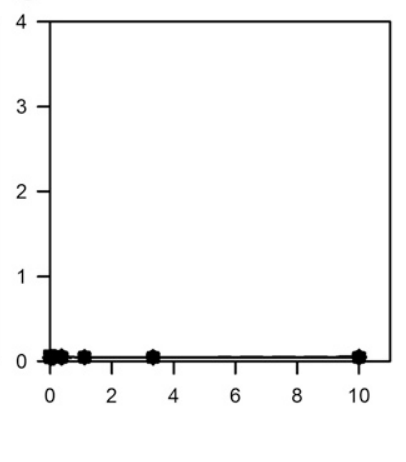

\section{$24 E 9 \mathrm{mAb}$ and $24 E 9 \mathrm{Fab}$ inhibits IE binding to ICAM-1 under} flow conditions

IE expressing DC4 PfEMP1 proteins adhere to ICAM-1 (12), a phenotype linked to sequestration of IE in the microvasculature of the brain $(6,35)$. We therefore tested whether the 24E9 Ab blocks this interaction. Biochips were coated with recombinant
ICAM-1, and 3D7 DC4 ${ }^{+}$parasites were flowed over at $1 \mathrm{dyn} / \mathrm{cm}^{2}$. The 24E9 mAb successfully inhibited adhesion at $1 \mu \mathrm{g} / \mathrm{ml}(67 \%$; $133 \mathrm{nM})$ and at $10 \mu \mathrm{g} / \mathrm{ml}(79 \% ; 1.3 \mathrm{mM})$, whereas the control mouse $\operatorname{IgG}(10 \mu \mathrm{g} / \mathrm{ml})$ failed to significantly alter adhesion to ICAM-1 (Fig. 4A). Fab fragments generated from 24E9 mAb were also assessed for inhibition at $1 \mu \mathrm{g} / \mathrm{ml}(83 \%$; $400 \mathrm{nM})$ and were
A
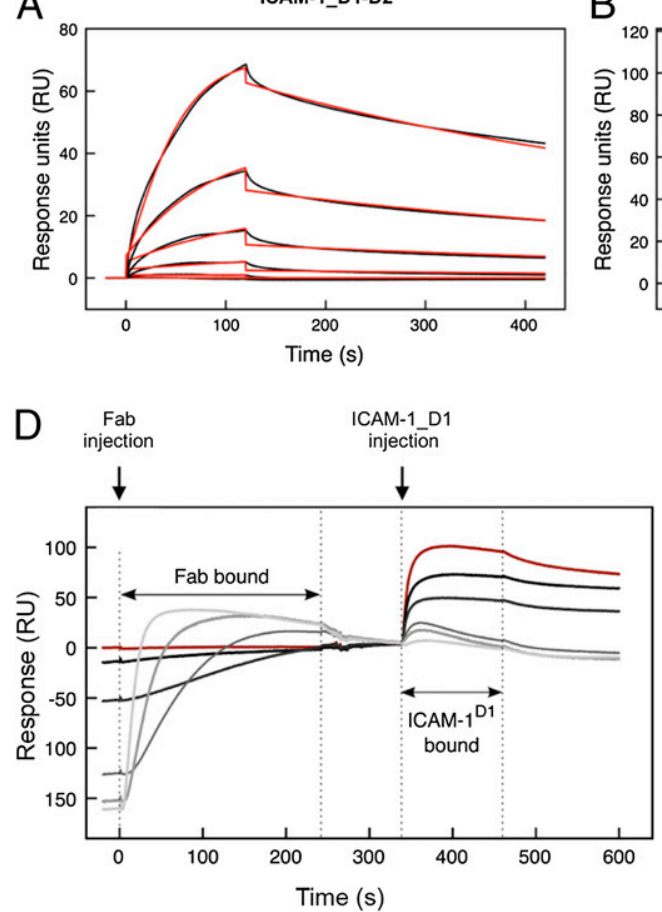
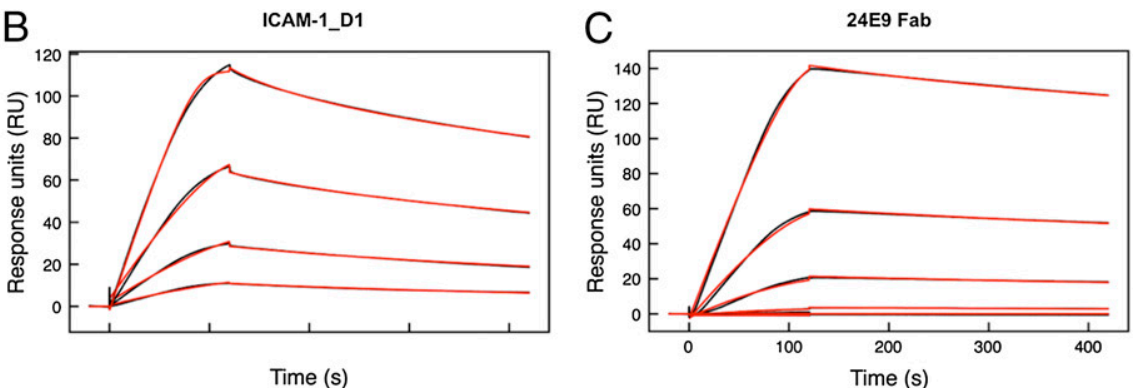

$E$

$\mathrm{F}$

FIGURE 3. 24E9 mAb inhibits ICAM-1 binding of DC4 DBLB3_D4. PFD1235w DBLB3_D4 was coupled to a sensor chip surface (RU = 400). Analytes were injected at $45 \mu \mathrm{l} / \mathrm{min}$ with an association phase of $120 \mathrm{~s}$ and a dissociation phase of $400 \mathrm{~s}$. Shown are sensorgrams for binding of DBL 33 _D4 to ICAM-1_D1-D2 (A), ICAM-1_D1 (B), and 24E9 Fab (C). Data (black lines) are modeled to a one-site model (red lines). (D) Sensorgrams observed for the sequential binding of 24E9 Fab fragment and ICAM-1_D1 to immobilized PFD1235w DBLB3_D4. Zero response is taken as the start of injection of ICAM-1_D1. A sensorgram for the binding of ICAM-1_D1 in the absence of 24E9 Fab is shown in red. (E) Quantification of the amount of ICAM-1_D1 binding to PFD1235w DBLB3_D4 after preincubation of the DBLB3_D4 with different concentrations of 24E9 Fab. (F) The ability of 24E9 mAb to inhibit DC4 DBLB3_D4 domains binding to ICAM-1-Fc as assayed by ELISA. Mouse IgG was added as control. Mean OD values are shown for three independent experiments. Error bars indicate SD. 
Table I. Kinetic parameters derived from SPR experiments on ICAM-1_D1-D2, ICAM-1_D1, and 24E9 mAb interacting with PFD1235w DBLB3_D4

\begin{tabular}{ccccc}
\hline \hline Interaction & $k_{\mathrm{a}}\left(\times 10^{5} \mathrm{M}^{-1} \mathrm{~s}^{1}\right)$ & $k_{\mathrm{d}}\left(\times 10^{-4} \mathrm{~s}^{-1}\right)$ & $K_{\mathrm{D}}(\mathrm{nM})$ & Model \\
\hline ICAM-1_D1-D2::DBLB3_D4 & 2.12 & 16.74 & 7.90 & One-site \\
ICAM-1_D1::DBLB3_D4 & 14.4 & 33.73 & 2.34 & One-site \\
24E9 Fab::DBLB3_D4 & 2.25 & 7.58 & 3.37 & One-site \\
\hline
\end{tabular}

titrated to determine the extent of activity. The Fab fragment continued to demonstrate adhesion inhibition at $>50 \%$ even at $0.001 \mu \mathrm{g} / \mathrm{ml}(67 \%$; $400 \mathrm{pM}$; Fig. 4A), whereas the control mouse $\mathrm{IgG} \mathrm{Fab}$ again failed to alter adhesion at the highest concentration tested $(1 \mu \mathrm{g} / \mathrm{ml})$. A second strain, BM57 $\mathrm{DC}^{+}$, was assessed, and like 3D7 $\mathrm{DC}^{+}$, adhesion was significantly inhibited by $24 \mathrm{E} 9 \mathrm{mAb}$ $(0.1 \mu \mathrm{g} / \mathrm{ml} ; 80 \%$ inhibition $)$ and 24E9 Fab $(0.001 \mu \mathrm{g} / \mathrm{ml} ; 83 \%$ inhibition) at the lowest concentrations tested (Fig. 4B). The specificity of adhesion to rICAM-1 was verified by preincubating control channels with anti-ICAM-1, which significantly reduced adhesion (81\% inhibition; Fig. 4).

\section{$24 E 9$ mAb recognizes a conformational epitope}

To determine whether 24E9 mAb interacts with a conformational epitope, we used Western blotting to test the reactivity of 24E9 $\mathrm{mAb}$ to reduced and nonreduced DBLB3_D4. As a control, we performed the same experiment with the non-DC4 PFD1235w DBLB3_D5 domain. 24E9 recognized only nonreduced DBLß3_D4 (Fig. 5A). We observed the same result by ELISA (Fig. 5B), where
24E9 recognized only nonreduced DBLß3_D4. To test whether the loss of reactivity of the mAb toward DTT-treated PFD1235W DBLB3_D4 was a result of the mAb being reduced in the ELISA, we performed the same assay using the PFD1235w DBL $\gamma$-specific human mAb (AB01), which is only partially dependent on the correct folding of DBL $\gamma$ (24). AB01 mAb was still able to recognize DTT-treated DBL $\gamma$ (Fig. 5C) showing that a similar mAb remained intact in the ELISA. This suggests that $24 \mathrm{E} 9 \mathrm{mAb}$ targets a conformational epitope.

The epitope targeted by $24 E 9$ partially overlaps with the potential ICAM-1 binding site of PFD1235w DBLB3_D4

To identify the specific peptides and surface features recognized by 24E9, we used HDX MS, a powerful, modern immunological method to examine epitopes bound by Abs under native conditions $(36,37)$. We analyzed the DBLß3_D4::24E9 Fab complex by measuring deuterium uptake over $200 \mathrm{~min}$ deuteration time for 83 partly overlapping peptides from DBLB3_D4, alone or in complex with 24E9 Fab. These correspond to $79 \%$ of the DBLB3_D4
FIGURE 4. 24E9 $\mathrm{mAb}$ and 24E9 Fab inhibit IE binding to ICAM-1 under flow conditions. Inhibition of adhesion by $24 \mathrm{E} 9 \mathrm{mAb}$ and $24 \mathrm{E} 9 \mathrm{Fab}$ of $3 \mathrm{D} 7 \mathrm{DC}^{+}$ (A) and BM57 DC4 ${ }^{+}$(B) to rICAM-1 coated onto Biochips. Abs were titrated at $0.1-10 \mu \mathrm{g} / \mathrm{ml}(24 \mathrm{E} 9$ $\mathrm{mAb}$ ) and $0.001-1 \mu \mathrm{g} / \mathrm{ml}$ (24E9 Fab). Each condition was run in triplicate for a minimum of three independent experiments and expressed as average number bound per square millimeter compared with untreated controls. Mouse $\mathrm{IgG}$ and mouse $\mathrm{IgG}$ Fab fragments were added as controls. Statistical significance was determined via one-way ANOVA with Tukey's multiple comparison test. $* * p<0.05, * * * p=0.0001$.
A

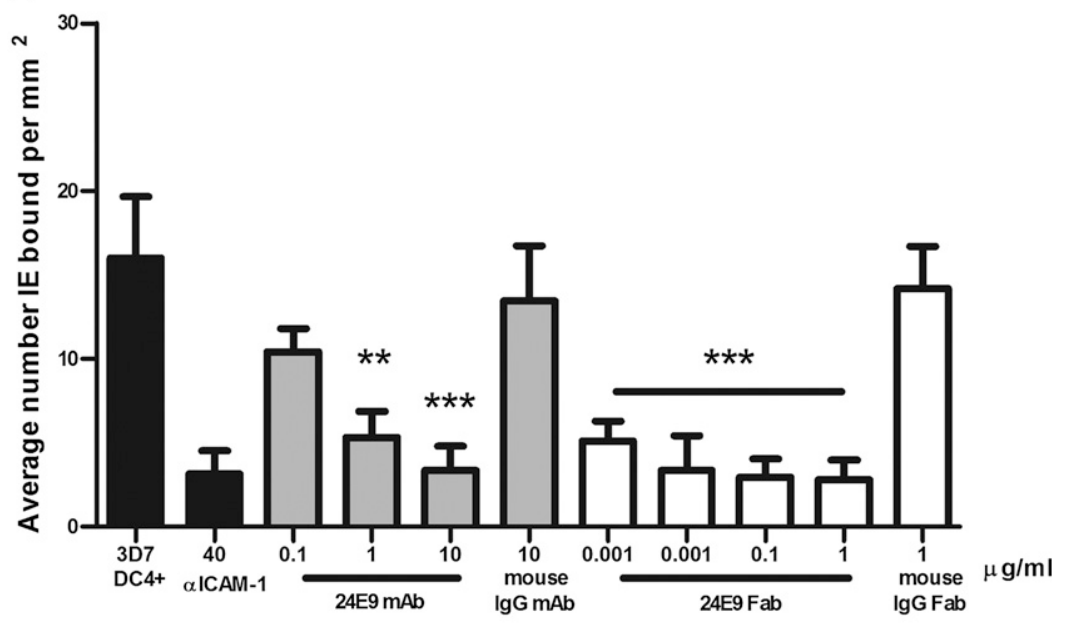

B

BM57 DC4+ Inhibition

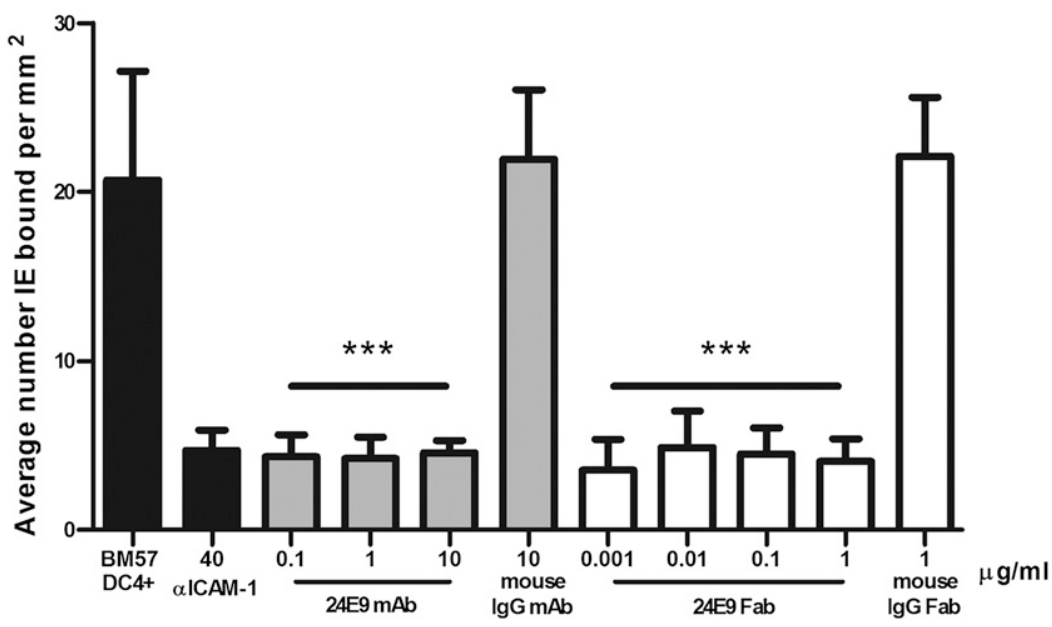



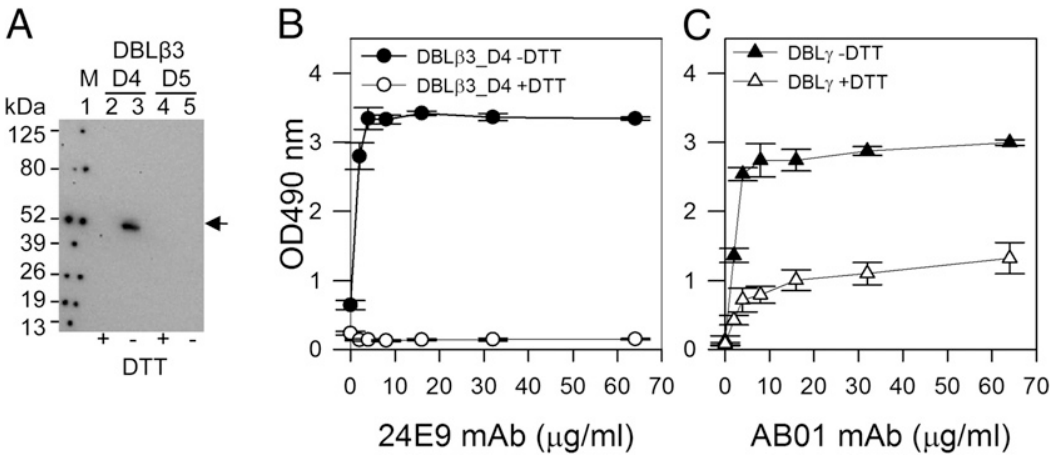

FIGURE 5. 24E9 mAb recognizes a conformation epitope on PFD1235w DBLß3_D4. (A) Western blotting of PFD1235w DBLß3_D4 (D4) and DBLB3_D5 (D5). +DTT (reduced), -DTT (nonreduced). Lane 1, Prosieve protein marker (M) visualized by phosphorescent paint as dots. Lanes 2 and 3 ,

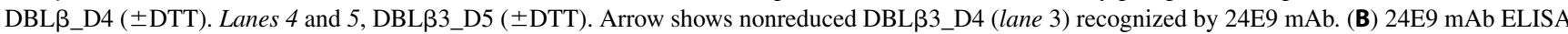
reactivity against reduced (+DTT) and nonreduced (-DTT) PFD1235w DBLß3_D4. (C) AB01 mAb ELISA reactivity against reduced (+DTT) and nonreduced (-DTT) DBL $\gamma$ of PFD1235w. Mean OD values are shown for three independent experiments. Error bars indicate SD.

primary sequence (Fig. 6A). Comparison of the level of deuteration highlighted three distinct regions, $\mathrm{P} 1, \mathrm{P} 2$, and $\mathrm{P} 3$, which show a reduction in deuterium uptake in the complex with $24 \mathrm{E} 9$ when compared with that of free DBLß3_D4 (Fig. 6A), indicating that these regions are masked by 24E9 Fab. Similar results were also observed for 20 min deuteration time (data not shown). P1 is located in the N-terminal third of DBLB3_D4 (subdomain 1), P2 in the center region (subdomain 2), whereas $\mathrm{P} 3$ is near the $\mathrm{C}$ ter-
A

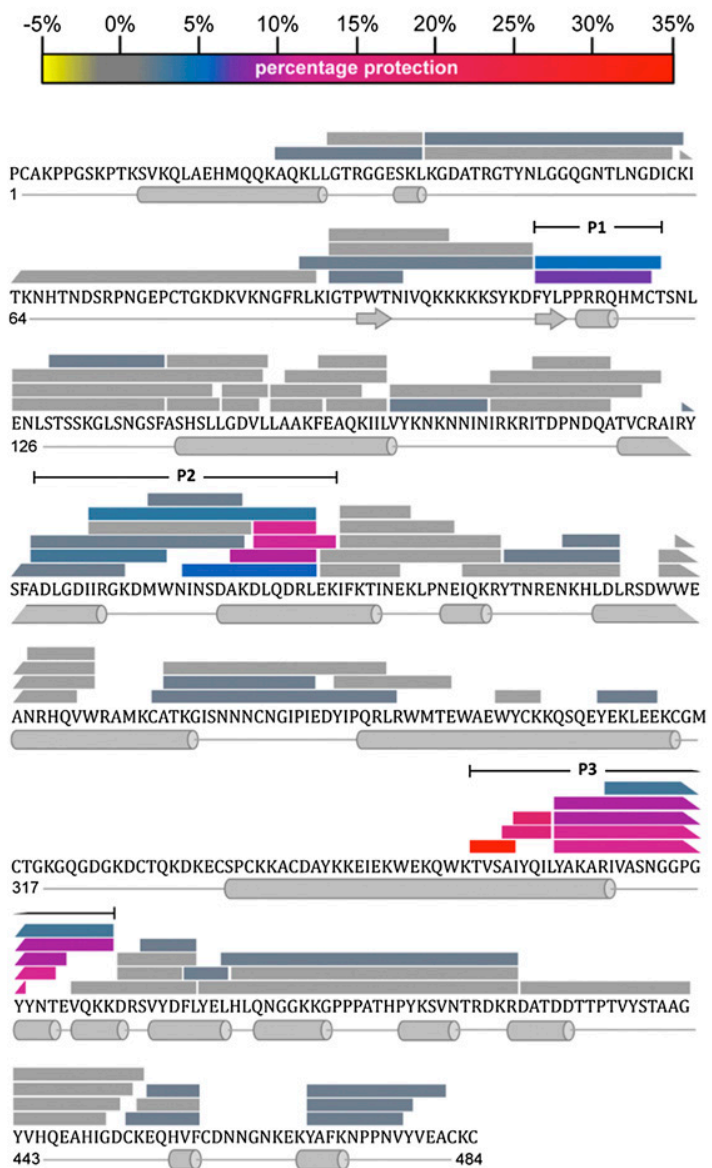

B
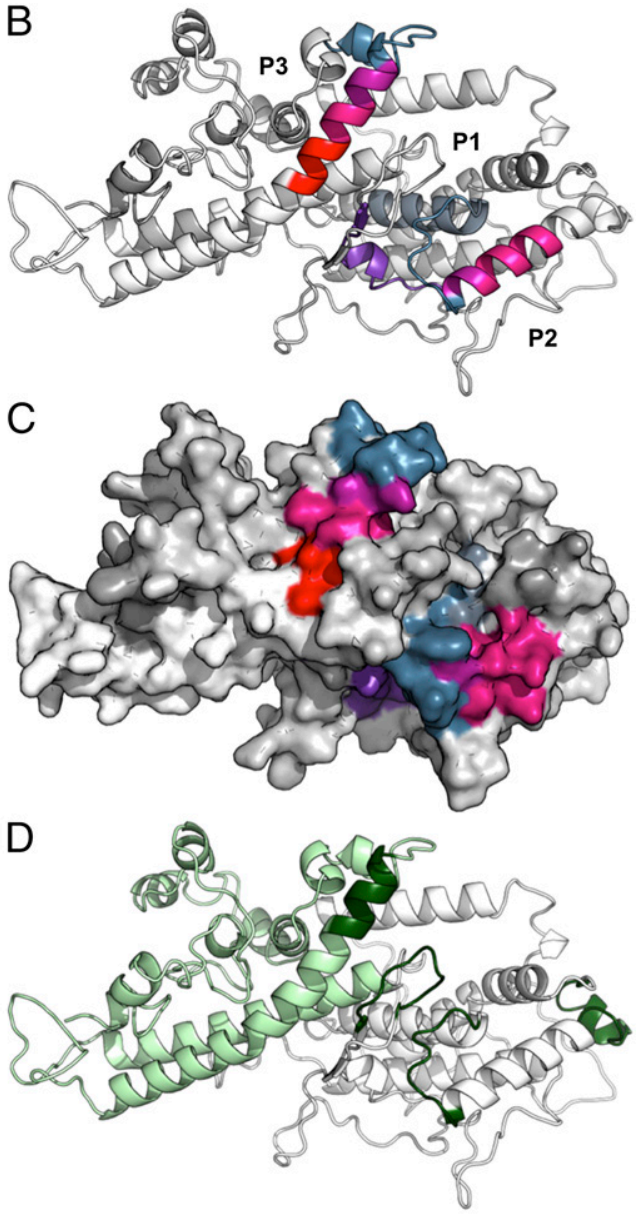

FIGURE 6. The 24E9 Fab binds to the convex surface of PFD1235w DBLß3_D4. (A) Peptides from DBLß3_D4 that were identified in mass spectra are represented by bars overlaying the primary sequence. The secondary structure, derived from a homology model of DBL $\beta 3$ _D4, is shown below the sequence. The level of protection of individual peptides, as determined by comparing the \%D incorporation over 200 min for free DBL $\beta 3$ D4 with that for DBLB3_D4 bound to 24E9 Fab, is color coded according to the scale bar. Highly protected areas are in red, whereas unprotected areas are in gray. Three highly protected regions were P1 (residues 110-121), P2 (193-220), and P3 (357-388), as indicated. (B) HDX MX results were mapped onto a model of the PFD1235w DBLB3_D4 domain. Protected areas are color coded as shown in (A). (C) Surface of PFD1235w DBL $\beta 3$ _D4 model as shown in (B). (D) Potential ICAM-1 binding sites on the DBLB3_D4 model as predicted by Bertonati and Tramontano (18) (green) and determined by Bengtsson et al. (12) (light green). 
A

Protein

PFD1235w DBLB3_D4

JF712900 DBL $\beta 3$

JF712901 DBLB3

JF712902 DBLB3

JF037695 DBL $\beta 3$

JF712903 DBL $\beta 3$

PFD1235W DBLB3_D5

D2dvar32 DBL $\beta 1$

IT4var13 DBL $\beta 5$

IT4var16 DBL $\beta 5$

B

PFD1235w DBLB3_D4 wt PFD1235w DBLB3_D4_P2b_D PFD1235w DBLB3_D4_P3a_D5
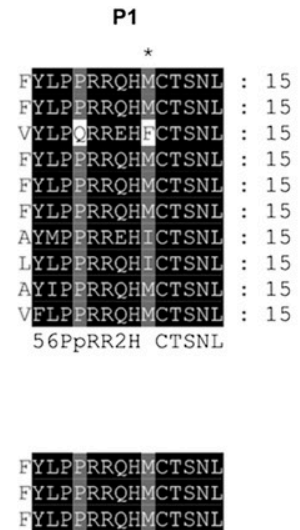

P2
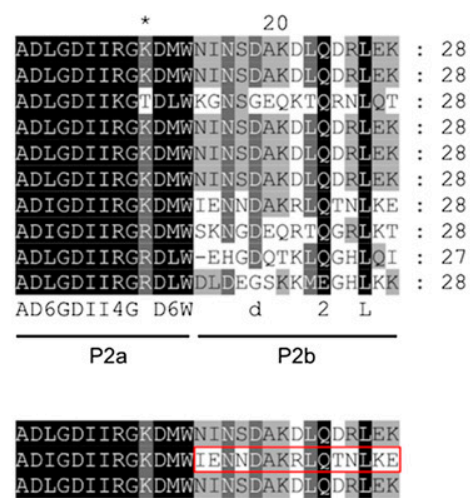

P3
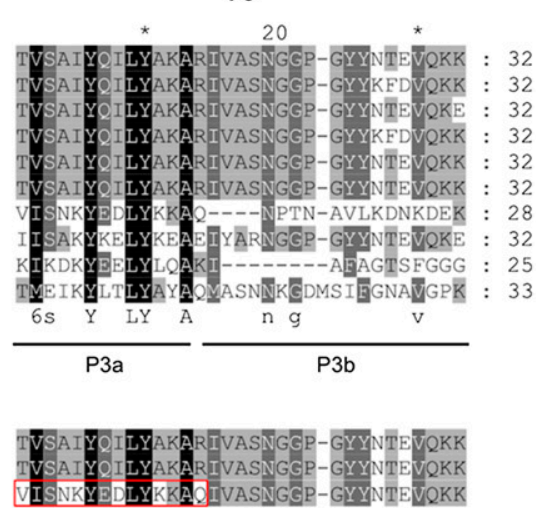
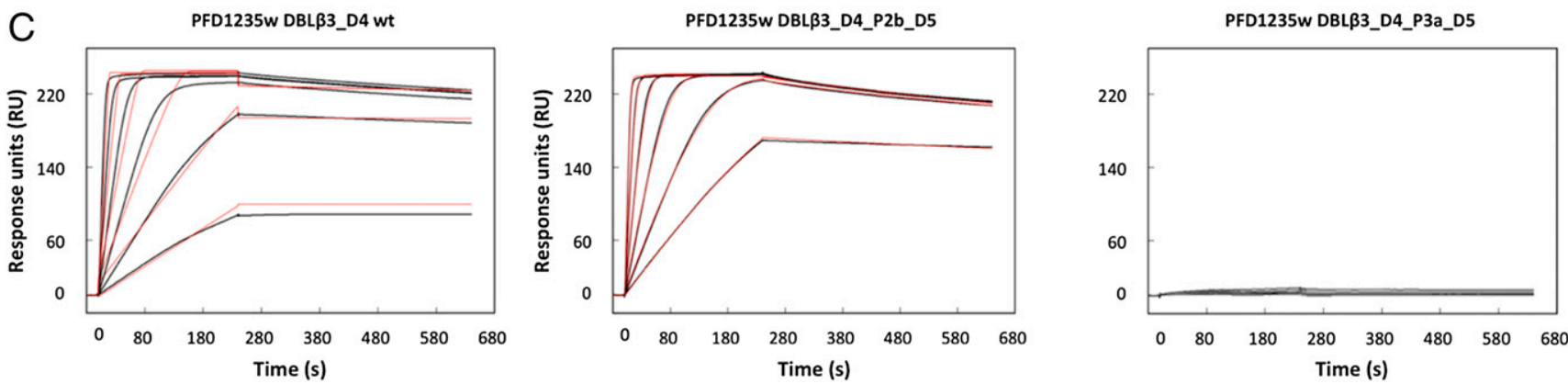

FIGURE 7. Fine mapping of the 24E9 binding site on DBL $\beta 3$ _D4. (A) Sequence conservation of P1, P2, and P3 among DBL $\beta$ domains. Sequences were aligned with MUSCLE. The ability of the DBL $\beta$ domains to bind to 24E9 is indicated. (B) PFD1235w DBL 33 D4 mutants were generated to determine the importance of protected peptides in the 24E9 mAb binding site. (C) SPR analysis of $24 \mathrm{E} 9 \mathrm{mAb}$ binding site mutants. 24E9 mAb was immobilized to 230 RU on a CM5 chip precoupled with protein G. Analytes were flowed over the chip surface at $40 \mu \mathrm{l} / \mathrm{min}$ with $240 \mathrm{~s}$ association and $400 \mathrm{~s}$ dissociation times. Data (black curves) were fitted to a one-site model (red curves).

minus of the protein and part of subdomain 3. When mapped on a homology model of DBLB3_D4, all three regions cluster to a well-defined, surface-exposed area (Fig. 6B, 6C), in accordance with the observation that 24E9 targets a conformational epitope (Fig. 5A-C). The size of this protected area is $2887 \AA^{2}$, which is comparable with the total $\sim 2800 \AA^{2}$ surface-exposed area of the variable loops of a Fab fragment.

The area protected by 24E9 Fab lies on the convex surface of DBL 33 D4. Mutational and modeling studies of non-DC4 DBL $\beta$ domains previously showed that this surface contains the ICAM-1 binding site $(18-20,38)$. Amino acids equivalent to residues important for the interaction between group B DBL $\beta$ domains and ICAM-1 (Fig. 6D, dark green) (18) partly overlap with P1, P2, and P3. Furthermore, the ICAM-1 binding site of DBLB3_D4 has been mapped to the C-terminal third of the domain (Fig. 6D, light green) (12). This includes region $\mathrm{P} 3$, which shows the strongest protection from deuteration in the DBLB3_D4::24E9 Fab complex. These observations indicate that the epitopes targeted by 24E9 overlap with the ICAM-1 binding site of DBLB3_D4.

To identify which of the protected peptides of DBLß3_D4 makes the most significant contribution to the binding affinity, we first compared the $\mathrm{P} 1, \mathrm{P} 2$, and $\mathrm{P} 3$ regions between DBL 33 domains that are recognized by 24E9 and those that are not (Fig. 7A). This revealed two motifs, P2b and P3a, which are mostly conserved only among 24E9 binding DBLB3 domains (Fig. 7A) and are strongly protected in the DBLß3_D4::24E9 Fab complex, suggesting that these motifs might directly contribute to 24E9 binding. To test this, we generated mutants by swapping the $\mathrm{P} 2 \mathrm{~b}$ and P3a peptides from DBLB3_D4 for the equivalent regions of the DBLB3_D5 domain (Fig. 7B), which is not recognized by 24E9. These mutants were expressed and purified as native DBLB3_D4, and their folding was confirmed by CD spectroscopy (Supplemental Fig. 1). The binding of the mutants to 24E9 mAb was analyzed by SPR, which showed that the exchange of the P2b peptide had little effect on 24E9 affinity, whereas exchange of the $\mathrm{P} 3 \mathrm{a}$ led to a complete loss of Ab binding (Fig. 7C, Table II). This demonstrates that P2 makes a minor contribution to 24E9 binding, whereas the P3 region contains an essential determinant of $\mathrm{Ab}$ binding.

\section{Low-resolution structure of the DBLB3_D4::24E9 Fab complex}

To understand better the architecture of the DBL 33 D4::ICAM-1 complex, we performed small-angle $\mathrm{x}$-ray scattering analysis of the DBLß3_D4 domain alone or in complex with 24E9 Fab (Fig. 8). The Rg determined from the composite scattering curve (Fig. 8A) was higher for the DBLß3_D4::24E9 Fab complex than for DBLB3_D4 alone (Table III). The increased Porod volume and

Table II. Kinetic parameters derived from SPR experiments on 24E9 mAb interacting with mutant version of PFD1235w DBL 33 _D4

\begin{tabular}{cccccc}
\hline \hline Interaction & $k_{\mathrm{a}}\left(\times 10^{7} \mathrm{M}^{-1} \mathrm{~s}^{-1}\right)$ & $k_{\mathrm{d}}\left(\times 10^{-5} \mathrm{~s}^{-1}\right)$ & $K_{\mathrm{D}}(\mathrm{pM})$ & $R_{\max }(\mathrm{RU})$ & Model \\
\hline DBL33_D4 wild type::24E9 $\mathrm{mAb}$ & 6.4 & 7.7 & 1.2 & 229.2 & One-site \\
DBL33_D4_P2b_D5::24E9 mAb & 1.87 & 4.1 & 2.19 & 239.2 & One-site \\
DBL33_D4_P3a_D5::24E9 mAb & 2.2 & 24 & 110 & 8.64 & One-site \\
\hline
\end{tabular}


A
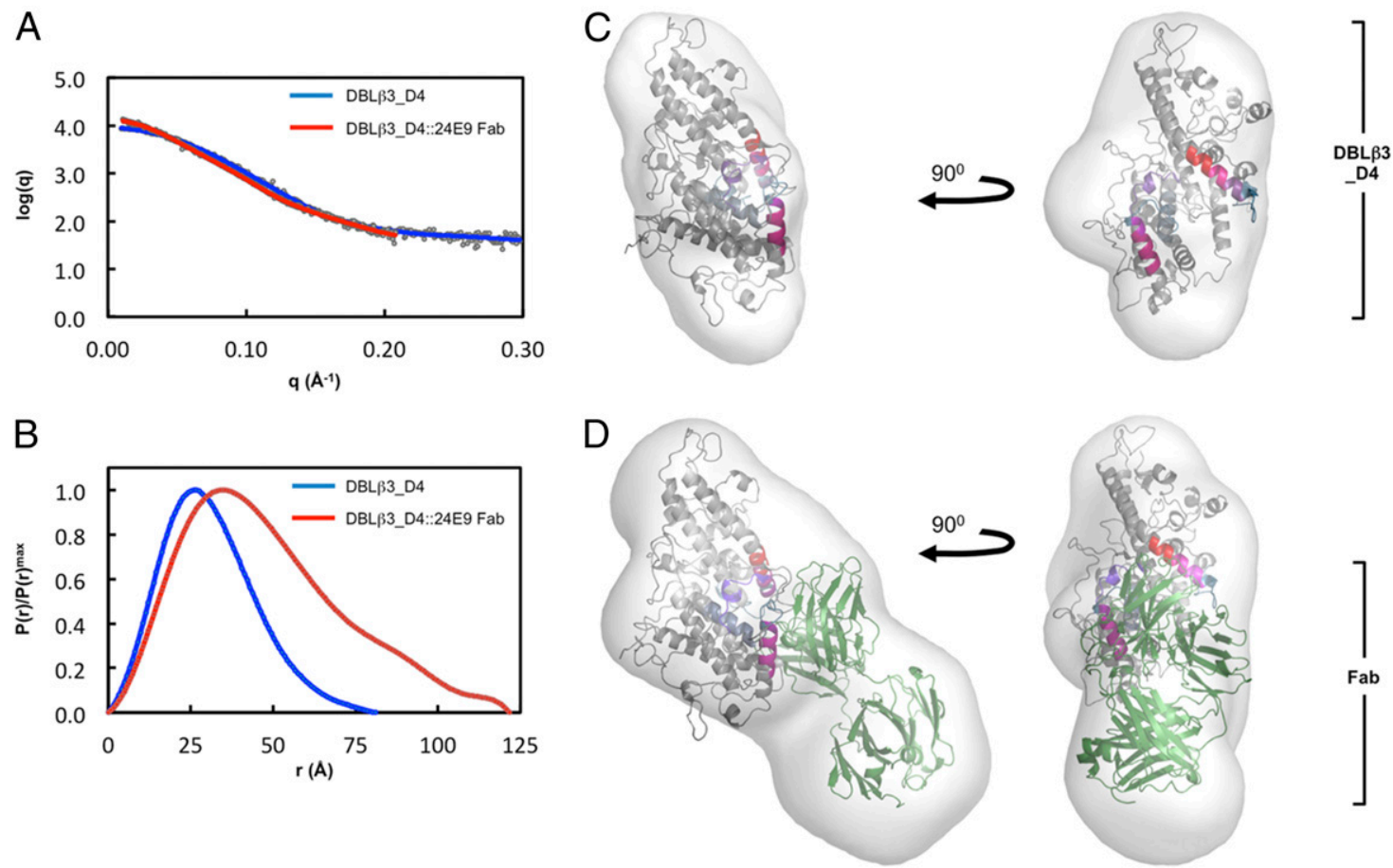

FIGURE 8. Molecular architecture of the PFD1235w DBL33_D4:24E9 Fab complex. (A) Theoretical scattering curves derived from ab initio models (solid lines) of DBL33_D4 and DBL33_D4::24E9 Fab superimposed on the experimental scattering data (circles). (B) Normalized distance distribution

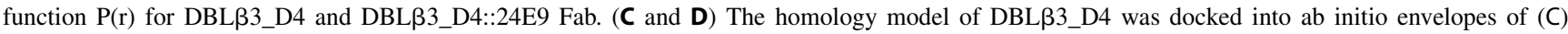
DBLB3_D4 alone and (D) the DBLß3_D4::24E9 Fab complex. The peptides identified as being protected in the DBL33_D4::24E9 Fab complex are color coded as in Fig. 5. The structure of a mouse Fab fragment (green, PDB ID 3GK8) was used as a model for 24E9 Fab.

the apparent molecular mass were also consistent with formation of a 1:1 complex between 24E9 Fab and DBLß3_D4 (Table III). The distance distribution function shows a more skewed profile for DBLß3_D4::24E9 Fab than that for DBLß3_D4 alone (Fig. 8B), indicating that binding of $24 \mathrm{E} 9 \mathrm{Fab}$ results in a more elongated particle (39). Accordingly, the $D_{\max }$ increases from $9.4 \mathrm{~nm}$ for DBL33_D4 to $12.2 \mathrm{~nm}$ for the complex (Table III).

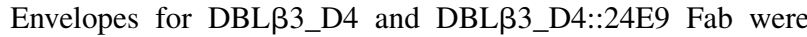
generated by ab initio modeling based on the scattering data, and the homology model of DBLB3_D4 was manually docked into these envelopes together with a model Fab fragment. Comparison of the two envelopes reveals that the DBL 33 _D4:24E9 Fab complex is more elongated, with an additional mass protruding from DBL33_D4, corresponding to 24E9 Fab (Fig. 8C, 8D). Simultaneous docking into this envelope positions the Ag binding loops of the Fab toward the regions identified as 24E9 binding site by HDX MS analysis (Figs. 6, 8D). This low-resolution shape reconstruction further supports the conclusion that 24E9 targets the convex surface of DBLB3_D4 and overlaps with its potential ICAM-1 binding site.

\section{Discussion}

The presence of inhibitory Abs that bind to the variable surface Ag PfEMP1 correlates with naturally acquired, protective immunity against PfEMP1-mediated IE adhesion during severe malaria (38, 40, 41). However, the epitopes targeted by such functional Abs and the mechanism by which they prevent IE adhesion are still unknown. In this study, we used immunological and biophysical methods to demonstrate that an $\mathrm{mAb}$ raised against a single DC4 DBL $\beta$ domain recognizes epitopes conserved between DC4 DBL $\beta$ domains and prevents ICAM- 1 binding by both purified domains and IE, occluding the ICAM-1 binding site on the surface of a DBL $\beta$ domain.

Of biological relevance, the 24E9 mAb and Fab fragments inhibit $3 \mathrm{D} 7 \mathrm{DC}^{+}$IE at picomolar to subnanomolar concentrations under physiological flow conditions (Fig. 4). Titration of the Abs not only confirmed the specificity of $24 \mathrm{E} 9$, but also illustrated how effective the $\mathrm{Ab}$ remained at low concentrations. Despite having only one Ag binding site, the 24E9 Fab is a more potent inhibitor of ICAM-1 binding than 24E9 mAb (Fig. 4A). This lower efficacy of the full-length and thus bulkier IgG molecule might be explained by steric hindrance and partially restricted access to the binding site of the native PfEMP1 as compared with the smaller Fab fragment. 24E9 also successfully inhibited the ICAM-1 adhesion of erythrocytes infected by a genetically distinct parasite, BM57 $\mathrm{DC}^{+}$. The $\mathrm{mAb} 24 \mathrm{E} 9$ is therefore able to inhibit ICAM-1 binding of DC4 DBLB3_D4 domains from a number of different parasite isolates, indicating that these domains share a common antigenic epitope.

Table III. Experimental values derived from PFD1235w DBLB3_D4::24E9 Fab SAXS experiments

\begin{tabular}{lcccccc}
\hline \hline & $\mathrm{Rg}_{\exp }(\mathrm{nm})$ & $D_{\max }(\mathrm{nm})$ & $\mathrm{V}_{\text {porod }}\left(\mathrm{nm}^{3}\right)$ & $\mathrm{Mr}_{\exp }(\mathrm{kDa})$ & $\mathrm{Mr}_{\text {app }}\left(\mathrm{kDa}^{2}\right)$ & \multicolumn{1}{c}{$\chi$} \\
\hline DBL33_D4 & 2.69 & 9.39 & 91.13 & 55 & 54.45 \\
DBL33_D4::24E9 Fab & 3.81 & 12.16 & 140.17 & 105 & 91.05 \\
\hline
\end{tabular}

The experimental $\mathrm{Rg}\left(\mathrm{Rg}_{\text {exp }}\right.$ ) was determined using AutoRG (8), the $D_{\max }$ was derived from GNOM (30), and the Porod volume (V $\mathrm{V}_{\text {porod }}$ ) was determined by using PRIMUS (29). The expected molecular mass $\left(\mathrm{Mr}_{\text {exp }}\right)$ is shown for DBLB3 D4 and the DBLB3 D4::24E9 complex. The apparent molecular mass (Mr ${ }_{\text {app }}$ ) was calculated from the volume excluded in the final DAMMIN (33) model divided by 2 . The $\chi$ value represents the best fit of 20 low-resolution shape reconstructions using ab initio modeling. 
Using HDX MS, we have identified three regions that cluster on the convex surface of PFD1235W DBLB3_D4 and that show reduced hydrogen-deuterium exchange in the presence of 24E9. One of these three peptides, the $\mathrm{P} 3 \mathrm{a}$ motif of region $\mathrm{P} 3$, was protected most strongly (Fig. 6) and is absolutely required for $\mathrm{Ab}$ binding (Fig. 7C). Indeed, this motif is strictly conserved only among DBL 33 domains recognized by 24E9. In addition, the P2b motif of region P2 is conserved in most 24E9-binding DBL 33 domains, but varies significantly between nonbinders (Fig. 7A). However, this motif plays only a minor role in 24E9 binding, as demonstrated by a slight reduction in $K_{\mathrm{D}}$ when it is mutated (Table II). In contrast, P1 and the remaining regions of P2 and P3 show a substantial degree of conservation between both 24E9-binding and nonbinding DBL $\beta$ domains (Fig. 7A), making it more likely that these amino acids are not part of the 24E9-binding site, but instead are sterically protected from hydrogen-deuterium exchange by the presence of the $\mathrm{Ab}$ binding to the neighboring epitopes. Our mapping data also suggest a mechanism by which 24E9 inhibits ICAM-1 binding, because epitopes recognized by mAb 24E9 cluster on the convex surface of DBL 33 D4 that is predicted to contain the binding site for ICAM-1 $(15,19,20,38)$. In addition, region $\mathrm{P} 3$, which contains the main determinant of 24E9 binding, lies within subdomain 3 of DBL 33 _D4. This subdomain forms a significant part of the convex surface of DBLB3_D4, and a previous study suggested that it is required for the interaction with ICAM-1 (12). These findings indicate that 24E9 exerts its inhibitory function by masking the ICAM-1 binding site of DBLB3_D4. These conclusions are further supported by our lowresolution shape reconstruction, determined by SAXS, which shows that 24E9 Fab adopts an orientation relative to the DBL 33 D4 that is similar to that of ICAM-1_D1-D2 bound to the DBL $\beta$ domain of IT4var13 (19).

The identification of the convex surface of DBL $\beta$ domains as the main target of inhibitory Abs provides important knowledge for choosing the components of a vaccine aimed at preventing PfEMP1-mediated adhesion of IE during severe malaria. A detailed mapping and structural characterization of the ICAM-1 binding sites of DBL $\beta$ domains from group A and B PfEMP1 and the identification of conserved surface features involved in this interaction are now needed to guide future decisions about how to design immunogens that elicit Abs inhibitory of ICAM-1 binding. Our observation that an $\mathrm{Ab}$ raised against a single DBL 33 D4 domain prevents the interaction between ICAM-1 and DBLB3_D4 domains from genetically distant parasite isolates demonstrates the existence of conserved antigenic epitopes. These might be used to specifically induce the production of Abs that cross-inhibit ICAM-1 binding by an important set of ICAM-1 binding DBL $\beta$ domains. Because DC4 DBL $\beta 3$ _D4 domains are found in group A PfEMP1, which have been associated with increased IE adhesion and severe malaria $(6,15,42)$, such conserved epitopes are promising candidates for inclusion in a vaccine that interferes with the PfEMP1::ICAM-1 interaction and confers strain-independent protection against severe malaria.

\section{Acknowledgments}

The authors thank Anina L. Jensen, Vera V. Pinto, Mette U. Madsen, Marianne A. Andersen, Michael B. Dalgaard, Kirsten P. Zimling, and Maiken H. Visti for excellent technical assistance. We acknowledge Maxime Rome from the Botanical Garden in Lyon for providing pitcher fluid from Nepenthes carnivorous plants, giving the opportunity to evaluate the activity of native nepenthesin in HDX MS conditions.

\section{Disclosures}

The authors have no financial conflicts of interest.

\section{References}

1. World Health Organization. 2014. World Health Organization, Geneva, Switzerland.

2. Hafalla, J. C., O. Silvie, and K. Matuschewski. 2011. Cell biology and immunology of malaria. Immunol. Rev. 240: 297-316

3. Kraemer, S. M., and J. D. Smith. 2006. A family affair: var genes, PfEMP1 binding, and malaria disease. Curr. Opin. Microbiol. 9: 374-380.

4. Smith, J. D., G. Subramanian, B. Gamain, D. I. Baruch, and L. H. Miller. 2000. Classification of adhesive domains in the Plasmodium falciparum erythrocyte membrane protein 1 family. Mol. Biochem. Parasitol. 110: 293-310.

5. Rask, T. S., D. A. Hansen, T. G. Theander, A. Gorm Pedersen, and T. Lavstsen. 2010. Plasmodium falciparum erythrocyte membrane protein 1 diversity in seven genomes-divide and conquer. PLOS Comput. Biol. 6: 6.

6. Turner, G. D., H. Morrison, M. Jones, T. M. Davis, S. Looareesuwan, I. D. Buley, K. C. Gatter, C. I. Newbold, S. Pukritayakamee, B. Nagachinta, et al. 1994. An immunohistochemical study of the pathology of fatal malaria. Evidence for widespread endothelial activation and a potential role for intercellular adhesion molecule-1 in cerebral sequestration. Am. J. Pathol. 145: 1057-1069.

7. Newbold, C., P. Warn, G. Black, A. Berendt, A. Craig, B. Snow, M. Msobo, N. Peshu, and K. Marsh. 1997. Receptor-specific adhesion and clinical disease in Plasmodium falciparum. Am. J. Trop. Med. Hyg. 57: 389-398.

8. Ochola, L. B., B. R. Siddondo, H. Ocholla, S. Nkya, E. N. Kimani, T. N. Williams, J. O. Makale, A. Liljander, B. C. Urban, P. C. Bull, et al. 2011. Specific receptor usage in Plasmodium falciparum cytoadherence is associated with disease outcome. PLoS One 6: e14741.

9. Marlin, S. D., and T. A. Springer. 1987. Purified intercellular adhesion molecule1 (ICAM-1) is a ligand for lymphocyte function-associated antigen 1 (LFA-1) Cell 51: 813-819.

10. Diamond, M. S., D. E. Staunton, A. R. de Fougerolles, S. A. Stacker, J. GarciaAguilar, M. L. Hibbs, and T. A. Springer. 1990. ICAM-1 (CD54): a counterreceptor for Mac-1 (CD11b/CD18). J. Cell Biol. 111: 3129-3139.

11. Joergensen, L., D. C. Bengtsson, A. Bengtsson, E. Ronander, S. S. Berger, L. Turner, M. B. Dalgaard, G. K. Cham, M. E. Victor, T. Lavstsen, et al. 2010. Surface co-expression of two different PfEMP1 antigens on single Plasmodium falciparum erythrocytes facilitates binding to ICAM1 and PECAM1. PLoS Pathog. 6: e1001083. Available at: http://journals.plos.org/plospathogens/article? id $=10.1371$ /journal.ppat. 1001083 .

12. Bengtsson, A., L. Joergensen, T. S. Rask, R. W. Olsen, M. A. Andersen, L. Turner, T. G. Theander, L. Hviid, M. K. Higgins, A. Craig, et al. 2013. A novel domain cassette identifies Plasmodium falciparum PfEMP1 proteins binding ICAM-1 and is a target of cross-reactive, adhesion-inhibitory antibodies. J. Immunol. 190: 240-249.

13. Jensen, A. T., P. Magistrado, S. Sharp, L. Joergensen, T. Lavstsen, A. Chiucchiuini, A. Salanti, L. S. Vestergaard, J. P. Lusingu, R. Hermsen, et al. 2004. Plasmodium falciparum associated with severe childhood malaria preferentially expresses PfEMP1 encoded by group A var genes. J. Exp. Med. 199: 1179-1190.

14. Smith, J. D., A. G. Craig, N. Kriek, D. Hudson-Taylor, S. Kyes, T. Fagan, R. Pinches, D. I. Baruch, C. I. Newbold, and L. H. Miller. 2000. Identification of a Plasmodium falciparum intercellular adhesion molecule-1 binding domain: a parasite adhesion trait implicated in cerebral malaria. Proc. Natl. Acad. Sci. USA 97: 1766-1771.

15. Chattopadhyay, R., T. Taneja, K. Chakrabarti, C. R. Pillai, and C. E. Chitnis. 2004. Molecular analysis of the cytoadherence phenotype of a Plasmodium falciparum field isolate that binds intercellular adhesion molecule-1. Mol. Biochem. Parasitol. 133: 255-265.

16. Springer, A. L., L. M. Smith, D. Q. Mackay, S. O. Nelson, and J. D. Smith. 2004. Functional interdependence of the DBLbeta domain and $\mathrm{c} 2$ region for binding of the Plasmodium falciparum variant antigen to ICAM-1. Mol. Biochem. Parasitol. 137: 55-64.

17. Mayor, A., N. Bir, R. Sawhney, S. Singh, P. Pattnaik, S. K. Singh, A. Sharma, and C. E. Chitnis. 2005. Receptor-binding residues lie in central regions of Duffy-binding-like domains involved in red cell invasion and cytoadherence by malaria parasites. Blood 105: 2557-2563.

18. Bertonati, C., and A. Tramontano. 2007. A model of the complex between the PfEMP1 malaria protein and the human ICAM-1 receptor. Proteins 69: 215-222.

19. Brown, A., L. Turner, S. Christoffersen, K. A. Andrews, T. Szestak, Y. Zhao, S. Larsen, A. G. Craig, and M. K. Higgins. 2013. Molecular architecture of a complex between an adhesion protein from the malaria parasite and intracellular adhesion molecule 1. J. Biol. Chem. 288: 5992-6003.

20. Howell, D. P., E. A. Levin, A. L. Springer, S. M. Kraemer, D. J. Phippard, W. R. Schief, and J. D. Smith. 2008. Mapping a common interaction site used by Plasmodium falciparum Duffy binding-like domains to bind diverse host receptors. Mol. Microbiol. 67: 78-87.

21. Bengtsson, A., L. Joergensen, Z. R. Barbati, A. Craig, L. Hviid, and A. T. Jensen 2013. Transfected HEK293 cells expressing functional recombinant intercellular adhesion molecule 1 (ICAM-1)-a receptor associated with severe Plasmodium falciparum malaria. PLoS One 8: e69999.

22. Owens, R. M., X. Gu, M. Shin, T. A. Springer, and M. M. Jin. 2010. Engineering of single Ig superfamily domain of intercellular adhesion molecule 1 (ICAM-1) for native fold and function. J. Biol. Chem. 285: 15906-15915.

23. Yokoyama, W. M., M. Christensen, G. D. Santos, D. Miller. 2006. Production of monoclonal antibodies. Curr. Protoc. Immunol. Chapter 2:Unit 2.5.

24. Barfod, L., M. B. Dalgaard, S. T. Pleman, M. F. Ofori, R. J. Pleass, and L. Hviid. 2011. Evasion of immunity to Plasmodium falciparum malaria by IgM masking of protective IgG epitopes in infected erythrocyte surface-exposed PfEMP1. Proc. Natl. Acad. Sci. USA 108: 12485-12490. 
25. Wang, Z., M. Raifu, M. Howard, L. Smith, D. Hansen, R. Goldsby, and D. Ratner. 2000. Universal PCR amplification of mouse immunoglobulin gene variable regions: the design of degenerate primers and an assessment of the effect of DNA polymerase $3^{\prime}$ to $5^{\prime}$ exonuclease activity. J. Immunol. Methods 233: $167-177$.

26. Nielsen, M. A., T. Staalsoe, J. A. Kurtzhals, B. Q. Goka, D. Dodoo, M. Alifrangis, T. G. Theander, B. D. Akanmori, and L. Hviid. 2002. Plasmodium falciparum variant surface antigen expression varies between isolates causing severe and nonsevere malaria and is modified by acquired immunity. J. Immunol. 168: 3444-3450.

27. Snounou, G., X. Zhu, N. Siripoon, W. Jarra, S. Thaithong, K. N. Brown, and S. Viriyakosol. 1999. Biased distribution of msp1 and msp2 allelic variants in Plasmodium falciparum populations in Thailand. Trans. R. Soc. Trop. Med. Hyg. 93: $369-374$

28. Kadek, A., V. Tretyachenko, H. Mrazek, L. Ivanova, P. Halada, M. Rey, D. C. Schriemer, and P. Man. 2014. Expression and characterization of plant aspartic protease nepenthesin-1 from Nepenthes gracilis. Protein Expr. Purif. 95 121-128.

29. Konarev, P. V., V. V. Volkov, A. V. Sokolova, M. H. J. Koch, and D. I. Svergun 2003. PRIMUS: a Windows PC-based system for small-angle scattering data analysis. J. Appl. Cryst. 36: 1277-1282.

30. Svergun, D. 1992. Determination of the regularization parameter in indirecttransform methods using perceptual criteria. J. Appl. Cryst. 25: 495-503.

31. Franke, D., and D. I. Svergun. 2009. DAMMIF, a program for rapid ab-initio shape determination in small-angle scattering. J. Appl. Cryst. 42: 342-346.

32. Volkov, V. V., and D. I. Svergun. 2003. Uniqueness of ab initio shape determination in small-angle scattering. J. Appl. Cryst. 36: 860-864.

33. Svergun, D. I. 1999. Restoring low resolution structure of biological macromolecules from solution scattering using simulated annealing. Biophys. J. 76: 2879-2886.
34. Birmanns, S., M. Rusu, and W. Wriggers. 2011. Using Sculptor and Situs for simultaneous assembly of atomic components into low-resolution shapes. $J$ Struct. Biol. 173: 428-435.

35. Silamut, K., N. H. Phu, C. Whitty, G. D. Turner, K. Louwrier, N. T. Mai, J. A. Simpson, T. T. Hien, and N. J. White. 1999. A quantitative analysis of the microvascular sequestration of malaria parasites in the human brain. Am. J. Pathol. 155: 395-410.

36. Abbott, W. M., M. M. Damschroder, and D. C. Lowe. 2014. Current approaches to fine mapping of antigen-antibody interactions. Immunology 142: 526-535.

37. Zhang, H., W. Cui, and M. L. Gross. 2014. Mass spectrometry for the biophysical characterization of therapeutic monoclonal antibodies. FEBS Lett. 588: 308-317.

38. Oleinikov, A. V., E. Amos, I. T. Frye, E. Rossnagle, T. K. Mutabingwa, M. Fried, and P. E. Duffy. 2009. High throughput functional assays of the variant antigen PfEMP1 reveal a single domain in the 3D7 Plasmodium falciparum genome that binds ICAM1 with high affinity and is targeted by naturally acquired neutralizing antibodies. PLoS Pathog. 5: e1000386.

39. Koch, D. I. S. 2003. Small-angle scattering studies of biological macromolecule in solution. Rep. Prog. Phys. 66: 1735.

40. Magistrado, P A. J. Lusingu, L. S. Vestergaard, M. Lemnge, T. Lavstsen, L. Turner, L. Hviid, A. T. Jensen, and T. G. Theander. 2007. Immunoglobulin G antibody reactivity to a group A Plasmodium falciparum erythrocyte membrane protein 1 and protection from P. falciparum malaria. Infect. Immun. 75: 24152420 .

41. Chan, J. A., K. B. Howell, L. Reiling, R. Ataide, C. L. Mackintosh, F. J. Fowkes, M. Petter, J. M. Chesson, C. Langer, G. M. Warimwe, et al. 2012. Targets of antibodies against Plasmodium falciparum-infected erythrocytes in malaria immunity. J. Clin. Invest. 122: 3227-3238.

42. Bull, P. C., M. Kortok, O. Kai, F. Ndungu, A. Ross, B. S. Lowe, C. I. Newbold, and K. Marsh. 2000. Plasmodium falciparum-infected erythrocytes: agglutination by diverse Kenyan plasma is associated with severe disease and young host age. J. Infect. Dis. 182: 252-259. 